\title{
Niña Mariela iqué tienen en común un Hogar y un Hospital?
} Pupil Mariela, what do an Orphanage and a Hospital have in common?

\author{
Ramiro Pérez Martín'
}

\section{Resumen}

En Argentina, la Ley Nacional de Salud Mental, $N^{\circ} 26.657$, del año 20 I0, indica disponer de un presupuesto determinado para realizar cambios particularmente significativos en las instituciones donde se internan pacientes por motivos de salud mental. Se promueve, en todo el país, la internación voluntaria o involuntaria en hospitales generales, a la vez que se anticipa el cierre de los hospitales especializados en salud mental. Sin embargo, algunas características demográficas y la marcada falta de accesibilidad a recursos especializados a lo largo del país permiten ubicar una fundada duda a dicha propuesta, aunque se dispusiera de los recursos indicados y, más aún, si se pretenden preservar los valiosos derechos que están en el espíritu de la Ley. Especialmente en lo que respecta a internaciones clínicamente involuntarias fuera de las grandes ciudades. El presente artículo se propone ilustrar y fundamentar clínicamente esta posición con el caso de la niña Mariela, en relación a pacientes de salud mental de todas las edades y criticar este aspecto de la Ley, señalando el riesgo para los usuarios de no crear sistemas de salud mental públicos de complejidad creciente accesibles a toda la población del país.

Palabras clave: Ley de Salud Mental argentina - Internación involuntaria - Hospital General - Escenarios federales - Salud Mental Pública.

\begin{abstract}
In Argentina, the National Mental Health Law (No. 26.657), from 2010, indicates that a specific budget is available to carry out particularly significant changes in the institutions where patients are admitted for mental health reasons. Voluntary or involuntary hospitalization in general hospitals is promoted throughout the country, while the closure of specialized mental health hospitals is anticipated. However, some demographic characteristics and the marked lack of accessibility to specialized resources throughout the country allow to locate a well-founded doubt to said proposal, even if the indicated resources were available and even more so, if it is intended to preserve the valuable rights that are in the spirit of the Law. Especially with regard to clinically involuntary hospitalizations outside the big cities. This article aims to illustrate and substantiate this position clinically -with the case of the girl Mariela-, in relation to mental health patients of all ages and to criticize this aspect of the Law, pointing out the risk for users of not creating increasingly complex public mental health systems accessible to the entire population of the country.
\end{abstract}

Keywords: Argentine Mental Health Law - Involuntary hospitalization - General Hospital - Federal scenarios - Public Mental Health

RECIBIDO I5/II/21 - ACEPTADO 12/12/21

1. Médico psiquiatra infanto-juvenil, psicoterapeuta, especialista en psicopatología y salud mental (IUSAM/APdeBA), especialista en pediatría.

Correspondencia:

rperezmartin@hotmail.com 


\section{Escenarios federales en Salud Mental}

Trabajar como psiquiatras en el campo de la Salud Mental nos ubica en escenarios de lo más diversos. Escenarios en los que, a la hora de planificar, resultará fundamental contemplar su accesibilidad a redes reales de recursos. Redes de aquellas que no se encienden con un click; redes de recursos humanos: vínculos laborales para acompañar mejor a los usuarios, si se quiere. Redes y escenarios que la pandemia por COVID-19, a excepción de la consolidación y delimitación de algunos aspectos no tan novedosos para la asistencia (tele-asistencia), en general, tornó aun más demandados, descarnados y solitarios.

Según el último Censo Nacional -sin contar las migraciones post-pandemia-, el 52,6\% de la población total del país vive en ciudades de menos de 500.000 habitantes (Censo Nacional, 2010). Aún en el marco de un profundo respeto por los Derechos Humanos, las necesidades de atención y los servicios que se pueden ofrecer en el campo de la Salud Mental no son los mismos en las grandes urbes que en pequeñas ciudades; en hospitales especializados (o sub-especializados) accesibles por autopista, que en hospitales generales sin especialistas y aislados de un sistema regional-provincial o nacional, o en centros de atención primaria a varias horas de ruta de ida y vuelta o por camino de tierra rural o montañoso, en el norte o en el sur de nuestro país; en el nivel de la prevención primaria o en situaciones agudas de internación, ante consultas desencadenadas por una coyuntura o en cuadros de larga evolución que requieren asistencia ambulatoria intensiva; en niños que en adolescentes, en adultos que en gerontes; en personas que cuentan con un grupo social o una familia (de origen, ampliada o adoptiva) o en aquellas que no, si es por sólo hacer mención a unas pocas variables que hacen a realidades clínicas tan diversas en nuestra clínica y en nuestro país. Escenarios federales muchas veces sabidos "de pasillo" pero sin estadísticas oficiales sobre Salud Mental que permitan planificar (y presupuestar) como sería deseable (o como dice la Ley).

\section{Historia de una internación involuntaria en un Hospital General alejado de las grandes ciudades}

La situación clínica que se presenta a continuación es la de una niña, a la que nombraremos Mariela, que nació casi con la Ley Nacional de Salud Mental (Congreso de la Nación Argentina, 2010), tiene 10 años.

Su primera década de vida trágica estuvo signada por el desamparo y el trato violento (maltrato psico- lógico, físico y sexual). Mariela sufrió un conjunto de "estresores" biológicos, psicológicos y sociales, que se abatieron sobre ella y que fueron, desde un marco de pobreza desde antes de su nacimiento, hasta recibir drogas de abuso por vía placentaria durante el período fetal, el haber sufrido repetidos abandonos de figuras primarias de crianza, la muerte de cuidadores cercanos y haber sido repetidamente testigo y víctima de violencia psicológica, física y sexual desde el nacimiento, hasta incluso la prostitución infantil a cambio de drogas para el adulto que la tenía entonces a su cargo. La sucesión de comparecencias a la Justicia como niña víctima posiblemente no hayan agotado los hechos sufridos. Así creció; sobrevivió, podríamos decir. No tan distinto a su madre biológica.

La historia de Mariela ilustra la situación de aquellos niños -en breve adolescentes y adultos- en que no parece haber ninguna situación vital que pueda ser significada como especialmente traumática (ni como especialmente saludable). Es decir, en estas historias de vida lo traumático no irrumpe bruscamente en una mínima secuencia de escenas apacibles -que se repiten o permanecen suficientemente- sino que cada vivencia es una pincelada más, sutil o grosera, en el cuadro de un paisaje pleno de terror. Huellas indelebles para un psiquismo en desarrollo, que dañan su cerebro, su subjetividad y su historia, obstaculizando soberbiamente el proceso de autonomía progresiva e inclusión social, y orientando, de un plumazo, underrotero, casi sin opciones, por el carril de la psicopatología grave de la infancia.

Algunas nosografías rescatan las consecuencias para el funcionamiento psíquico o describen los antecedentes y comportamientos de vidas como la de Mariela, con cuadros ya definidos previamente como Patologías Límite, en diversas versiones de la Clasificación Francesa de Trastornos Mentales del Niño y del Adolescente (Asociación Franco-Argentina de Psiquiatría y Salud Mental, 2004; Fédération Française de Psychiatrie, 2017; Golse et al., 2020) o con el flamante Trastorno por Estrés Postraumático Complejo, de la 11va. Clasificación Internacional de Enfermedades (Organización Mundial de la Salud, 2019), respectivamente. Parafraseando a Winnicott en su idea de salud y de trauma, podría decirse que, a pesar de su tendencia innata al desarrollo, la infancia de Mariela se forjó a partir de permanentes rupturas de la continuidad en la línea de su existencia, en hechos o circunstancias más o menos sutiles (Winnicott, 2011).

Las situaciones que se relatan a continuación ocurrieron antes del inicio de la pandemia por CO- 
VID-19, en una ciudad de menos de 100.000 habitantes alejada de grandes centro urbanos, en el interior de una provincia argentina. Una localidad con demografía y accesibilidad a recursos especializados en Salud Mental escasos o inexistentes como infinidad de otras de la Argentina.

La madre de Mariela, a sabiendas de su propia historia, entrega a su hija al Estado, entre otros motivos, para que no tenga la misma vida que ella. El Estado ofrece a Mariela la única institución que tiene a su alcance en el distrito, un Hogar Convivencial para niños. Allí, por ser el tipo de Hogar de menor complejidad, no debería haber otros niños con cuadros psicopatológicos importantes, ya que no es un Hogar Especializado en Salud Mental (Hogar Terapéutico). En ese caso -nos informarían luego-, necesitaría una derivación formal a una gran ciudad, lejos de su tierra natal y de sus escasos vínculos. Si fuera adolescente, no se sabría dónde ubicarla para preservar sus derechos ya que cerraron el único hogar convivencial (con granja) que había en la región. Mucho menos si cumpliese los 18 años o si tuviera alguna discapacidad orgánica. Todavía, cada tanto, y a pesar de la Ley Nacional de Protección Integral de Derechos de Niñas, Niños y Adolescentes No 26.061 (Congreso de la Nación Argentina, 2005), surgen desveladas intenciones de cerrar Hogares en distintos lugares del país como modo de salvar a los niños del mundo cruelmente humano y desigual en que vivimos. Sin embargo, nunca se repensó el funcionamiento de muchos Hogares, ni terminaron de funcionar plenamente otras modalidades de alojamiento para niñas, niños o adolescente vulnerados. Solo por mencionar un ejemplo, en cualquier Jardín de Infantes o Escuela Primaria, el Estado recibe a cualquier alumno con una persona con formación terciaria específicamente orientada a las necesidades de un niño, e incluso a la detección precoz de patologías prevalentes. Sin embargo, en un Hogar convivencial promedio del interior del país, que hace las veces de familia y vivienda sustituta de niños con vidas traumatizadas y derechos elementales ya vulnerados, lo recibe muchas veces un grupo de cuidadores que, en el mejor de los casos, tiene buenas intenciones y ganas, pero al que no se le exige ningún tipo de formación o capacitación específica, ni ningún requisito educativo o atraviesa un proceso cuidadoso de selección. En ocasiones, ese tipo de lugares hasta puede llegar a ser depositario de personal "conflictivo" en otros ámbitos. A Ley "nueva”, funcionamiento viejo. ¿Los niños primero? Vemos.
Mariela comienza un tratamiento psicológico, pero la llevan a ver a su terapeuta esporádicamente "por temor a que se escape", y también la atiende un profesional médico sin especialidad en psiquiatría ni en psiquiatría infanto-juvenil. La función del estimado colega, sin capacidad para complejizar su mirada y su intervención clínica, es acotada y solo indica psicofármacos al cabo de los escasos minutos en los que logra sostener la presencia de Mariela en la consulta. El comprometido abordaje de la psicóloga psicoterapeuta no alcanza a operar en todos los frentes necesarios. El vínculo de Mariela con el personal del Hogar se desgasta cada vez más. La niña entra en una espiral de conductas (¿síntoma institucional, síntoma del sistema?) que la ponen claramente en riesgo a ella y a todos sus compañeros del Hogar. Desde ya, ni el edificio de la institución ni su personal están preparados para contener una situación como la que se desencadenó, y entonces el colega médico no especializado indica la internación de la paciente en el único Hospital General de la pequeña ciudad en la que se desarrolla el drama. Los responsables del Hogar rechazan formalmente su reingreso. Mariela se interna en la Sala de Pediatría -al lado de la Maternidad-, donde el único pediatra de guardia se ocupa de la guardia interna (lo que incluye la internación pediátrica general, terapia pediátrica, terapia neonatal, la recepción neonatal en partos y, en ocasiones, colaborar con el pediatra de la guardia externa). Ahora también este profesional debe ocuparse de la situación de Mariela. Ningún profesional del muy acotado equipo de salud mental polifuncional (con o sin especialidad) está las 24 horas en el Hospital. Además de haber jaqueado al subsistema de Desarrollo Social (Hogar), Mariela comienza a poner en jaque otro subsistema estatal paradójica e históricamente frágil: el de Salud Mental.

Luego de un conflicto laboral -estando Mariela aún internada y descompensada- el colega no especializado decide renunciar y otro, en similar situación, se aparta del caso por no ser de su incumbencia por la edad. De esta manera, incluso sin ser personal del hospital, se me convoca desde la Secretaría de Salud (no hay Secretaría de Salud Mental) como psiquiatra infanto-juvenil para hacerme cargo del caso de Mariela. Aun a sabiendas del escenario que debía enfrentar: el desamparo de una ya conocida Mariela y la urgencia - "esa tramposa" - de un sistema proclive al incendio; impulsado por esa suerte de "subjetividad heroica" a la que tendemos a ser adeptos los trabajadores de la salud mental -y de la salud en general-, acepté el convite a jugar de "bombero" (De la Aldea, 2014). 
Junto con la psicóloga comenzamos a intervenir. A la par de un tratamiento psicofarmacológico y psicoterapéutico, y con el fin de colaborar en sostener el desborde corporal de Mariela, darle lugar a su propia subjetividad, reconstruir su lugar de niña y promover el sostenimiento de lazos saludables con pares, su desarrollo emocional y su autonomía progresiva, se improvisaron con enorme esfuerzo, de al menos una veintena de personas, talleres diarios con aspectos lúdicos, artísticos, ocupacionales, se incluyó lo escolar, el ejercicio físico recreativo, salidas a un patio del hospital, etc. Si bien Mariela lograba por momentos participar activa y productivamente de estos talleres y espacios, la continuidad y desarrollo de los mismos se vieron marcadamente afectados por la ausencia de un equipo capacitado y experimentado en abordajes terapéuticos complejos en salud mental y por las características edilicias no adecuadas para internaciones de salud mental.

En relación al recurso humano con el que se contó en esta internación, se presentaron importantes obstáculos a la hora de intervenir y pensar conjuntamente, tanto con profesionales internos como externos al hospital (sin especialización ni capacitación en salud mental o salud mental infanto-juvenil).

El equipo del Hogar dejo de intervenir a partir de la internación, por lo que, desde el municipio, se organizó un equipo de cuidadores externos las 24 horas (provenientes del "Banco de Tareas" municipal, constituido por personas con escasos recursos económicos con las cuales el municipio colabora, abonando algún servicio y solicitándoles, como contraprestación, diversas tareas). Luego de pocos días, estos cuidadores abandonaron la tarea agotados por la misma, por lo que el Área de Enfermería del hospital coordinó horas extras de todo su plantel para ocuparse del acompañamiento de Mariela. Una parte de los miembros de ese plantel, agotados e impotentes, también fue abandonando la tarea y, en función de sostener la internación y evitar situaciones de autoagresión de la niña, se terminó convocando a personal femenino de la comisaría local. Hecho habitual, lamentable y naturalizado (¿ilegal?) en internaciones de salud mental involuntarias, particularmente en lugares alejados de grandes centros urbanos, que pretenden, paradójicamente, resguardar el derecho a la salud y a la vida (¿un retorno a antes de Pinel?). La angustiosa soledad ante la franca carencia de instalaciones edilicias y de un equipo de profesionales de salud mental ultraespecializados en soportar, contener y encausar desbordes psíquicos que amenazan la vida propia y/o ajena del paciente, derivan en intervenciones bienintencionadas o heroicas, pero de bordes filosos. Además, durante todo el transcurso de la internación e incluso en las actividades educativas, terapéuticas y de talleres que se intentaron organizar para la niña, ésta no mantuvo interacción con pares. Tampoco resultó posible regular el ingreso de objetos ni personas de la comunidad que, al tanto de la situación de la niña por las redes sociales, se acercaban a aportar sus buenas intenciones sin iguales efectos.

La cama de Mariela estaba en una habitación con otras camas de internación pediátrica (anuladas al efecto de dar espacio a la paciente) y un baño, que obviamente contaba con tomacorrientes comunes, teclas de luz, bocas de oxígeno y de aspiración, ventanas de vidrio común, sin medidas de seguridad hacia el exterior y espejos, por mencionar solamente las principales deficiencias de seguridad. Durante su estadía, la habitación se encontraba a oscuras, únicamente con luz artificial, con la persiana baja "por temor a que se escape", con los riesgos implícitos que tal situación conllevaba. Obviamente, el edificio del hospital no está preparado y no es arquitectónicamente seguro ni funcional para ese tipo de internaciones. Más allá de lo arquitectónico, el espacio físico en una internación de salud mental (encuadre) cumple, además, una función en la restitución del equilibrio mental que muchas veces es pasado por alto.

\section{Recursos humanos y edilicios especia- lizados: ¿castigo para el usuario, heroís- mo profesional o salud mental pública de avanzada?}

Por todo lo descrito, y luego de un mes de internación, se consideraron totalmente agotados todo tipo de recurso edilicio y humano disponibles, que permitieran sostener adecuadamente esta intervención de salud mental para Mariela, con los mínimos márgenes de seguridad y de trabajo posible del equipo terapéutico en salud mental, necesarios para orientar la intervención y respetando los más básicos derechos humanos, consignados en la Ley Nacional de Salud Mental $\mathrm{N}^{\circ}$ 26.657 así como en la de Derechos del Niño N² 26.061 y sus correspondientes leyes provinciales. Ante un episodio en que Mariela, aún bajo el cuidado de una mujer policía, intentó autolesionarse en su habitación con la corriente eléctrica y considerando que un Hospital especializado en Salud Mental Infanto-Juvenil de la Ciudad de Buenos Aires dispone del recurso edi- 
licio y humano necesarios, solicité la derivación al mismo, con el único apoyo del Juzgado de Familia y la Asesoría de Menores intervinientes, que lo autorizaron judicialmente.

En el Hospital especializado (mal llamado monovalente en la jerga sanitaria argentina), Mariela requirió de sólo dos semanas para estar en condiciones clínicas de egresar de su internación. Curiosamente, lo hizo con el mismo diagnóstico, similar propuesta de abordajes terapéuticos integrales y prácticamente el mismo esquema de tratamiento psicofarmacológico: ¿cuál fue la diferencia? No fue un "héroe" ni un grupo enorme de bienintencionados quienes lo lograron; fueron recursos humanos y edilicios especializados y respetuosos de los Derechos Humanos. Desde ya, se le podrán criticar diversas cosas a los Hospitales Especializados, pero difícilmente que dejen en la calle a un paciente en situación de extrema vulneración mental.

Mariela ilustra, lastimosamente, como se reempla$\mathrm{za}$, en el interior profundo del país, el desamparo de un sistema de salud mental inexistente y, peor aún, como parece que se reemplazaría un hospital especializado en salud mental con un hospital general, aún frente al más avanzado, prolífico y necesario desarrollo de la salud mental comunitaria y la prevención primaria en salud mental.

Su condición de niña realza la importancia de la situación de Mariela como caso índice para todas las edades de la salud mental, por la mayor prevalencia de internaciones en la adolescencia o adultez y porque enciende las alertas ante la lisa y llana ausencia de un sistema de salud mental, nacional, provincial o municipal, fuera de las grandes ciudades.

La patología mental, con sus variables biológicas, psicológicas y socio-históricas, demuestra en las más diversas partes del mundo, que su existencia no es arbitraria ni respeta reduccionismos ni límites demográficos de ningún tipo; y la pandemia por COVID-19 recordó al mundo entero la importancia de priorizarla. Incluso a los mismos organismos internacionales que, obnubilados de teoría, promueven cierres de instituciones, en lugar de un sistema de salud mental pública de avanzada y con complejidad creciente; con salvaguarda de los Derechos Humanos, con Promoción y Prevención Primaria de la Salud, con Salud Mental Comunitaria, con Hospitales Generales y con Hospitales especializados que, entre otras funciones, sepan y puedan contener internaciones involuntarias de todas las edades, distribuidos en todo el país.

Creer que todo hospital general -en cualquier lugar del país- puede estabilizar, en una internación de salud mental, cuadros graves y descompensados en pacientes sin conciencia de situación (internaciones clínicamente involuntarias, más allá del requisito legal de involuntario a todo niño/a), no sólo parece una mirada groseramente ingenua o negacionista de la patología mental sino que también parece una mirada altamente riesgosa y reduccionista. Ésta última, supone que estabilizar un paciente dependería, médicamente, de sólo "dar en la tecla" con la medicación, desconociendo la dimensión humana y psicoterapéutica de la asistencia en psiquiatría (¿el meollo de la especialidad?) y de todo acto médico. Sumado a ello, se desconoce la experiencia de todo el personal de un hospital especializado (profesionales de todas las disciplinas, áreas y rangos, acompañantes terapéuticos, talleristas, docentes, personal de maestranza, de cocina, porteros, etc.) en el manejo de situaciones específicas y complejas, además de la cuestión edilicia y de la brutal inexistencia de psiquiatras y psiquiatras infanto-juveniles distribuidos razonablemente en la amplia geografía del país.

La lucha contra los reduccionismos y en favor de los derechos de las personas con padecimiento o patología mental se da en las universidades, residencias, asociaciones y gremios; la lucha contra el estigma en salud mental se da también en lo cotidiano, lo familiar, en los barrios, los consultorios, las escuelas, los ámbitos laborales; nada de esto se gana por sí solo en las salas de internación de los hospitales; tampoco en los Hogares. Visto desde estos lares, ¿será la derivación de Mariela un resabio de la medieval pena de destierro por "mala conducta social"? (Asenjo González, 2014).

A más de 10 años de la aún controvertida Ley 26.657 -en aspectos, no en espíritu- y en camino a los 10 años de su reglamentación (2013), quizá tenga más impacto poblacional lo que hay por desarrollar en materia de salud mental, que lo que hay por adecuar $\mathrm{u}$ optimizar -no cerrar- pero esto pertenece al ámbito de las opiniones personales.

Incluso con la pandemia por COVID-19 aún a cuestas y sus efectos en la salud mental aún por verse, cabría preguntarse qué aprenderíamos para el conjunto si miráramos las partes con otros lentes; cabría preguntarle a la niña Mariela por el funcionamiento de los hogares, por el sentido de la división en convivenciales y especializados.

Qué respondería Mariela si le preguntáramos ¿qué tienen en común la sensación de desamparo en un Hogar y en un Hospital General del interior del país?, ¿no necesitamos más especialistas y más lugares es- 
pecializados en el país para ayudarte? Y nosotros preguntarnos ¿cuáles son los riesgos de cerrar hospitales especializados para los distintos escenarios de la salud mental en la Argentina?

Todas preguntas acuciantes que requieren respuestas urgentes.

Conflicto de intereses: el autor declara no presentar conflicto de intereses.

Agradecimientos: a Mariela, por permitirnos aprender a partir de su sufrimiento.

\section{Referencias bibliográficas}

Asenjo González, M. (2014). La exclusión como castigo. La pena de destierro en las ciudades castellanas del siglo XV. Anales de la Universidad de Alicante. Historia Medieval, 18, 63-93. https://doi.org/10.14198/medieval.2012-2015.18.02

Asociación Franco-Argentina de Psiquiatría y Salud Mental. (2004). Clasificación Francesa de los Trastornos Mentales del Niño y del Adolescente (CFTMEA-R-2000) Polemos.

Censo Nacional (s/d). Población 2010. (Último acceso: 6 de noviembre de 2020). https://www.ign.gob.ar/NuestrasActividades/Geografia/DatosArgentina/Poblacion2
Congreso de la Nación Argentina. (s/d). Ley Nacional de Protección Integral de los Derechos de Niños, Niñas y Adolescentes (No 26.061). (Último acceso: 8 de noviembre de 2020). http://servicios.infoleg.gob.ar/infolegInternet/anexos/110000-114999/110778/norma.htm

Congreso de la Nación Argentina. (s/d). Ley Nacional de Salud Mental (No 26.657). http://servicios.infoleg.gob.ar/infolegInternet/anexos/175000-179999/175977/norma.htm

(Último acceso: 12 de noviembre de 2020).

De la Aldea, E. (2014). La subjetividad heroica: Un obstáculo en las prácticas comunitarias de la salud. Los Talleres - Cuidar al Que Cuida, 1(1), 7-26. Fédération Française de Psychiatrie. (2017). Clasificación francesa de los trastornos mentales del niño y del adolescente R-2012 (5ta. Edición). Clasificación francesa de los trastornos mentales del adulto R-2015 (1ra edición). Polemos.

Golse, B., Botbol, M., Bursztejn, C., \&Portelli, C. (Eds.). (2020). Classification française des troubles mentaux de l'enfant et de l'adolescent-R-2020. Classification psychopathologique et développementale. (6ta. edición). Presses de l'Ecole des Hautes Études en Santé Publique.

Organización Mundial de la Salud. (2019). Herramienta de codificación de la CIE-11. Clasificación Internacional de Enfermedades, 11a. revisión - estandarización mundial de la información de diagnóstico en el ámbito de la salud. https://icd.who.int/ct11/icd11_mms/es/release (Último acceso: 8 de noviembre de 2020).

Winnicott, D. W. (1967). El concepto de individuo sano. En El hogar, nuestro punto de partida: Ensayos de un psicoanalista (p. 336). Paidós. 